\title{
El uso de Antiiflamatorios no esteroideos disminuyó el riesgo de enfermedad de Alzheimer
}

Nonsteroidal antiinflammatory drugs and the risk of Alzheimer's disease. Veld BA, Ruitenberg A, Hofman A et al. N Engl J Med 2001; 345: 1515-1521.

\section{Objetivo}

Determinar si el uso de antiinflamatorios no esteroideos (AINES) está asociado o no con la disminución de riesgo de adquirir Enfermedad de Alzheimer (EA) o demencia vascular (DV).

Diseño

Estudio de cohorte*, prospectivo, conducido entre 1991 y 1998 . El riesgo de desarrollar EA fue estimado en relación al uso de AINEs documentados en un registro de farmacias (The Rotterdam Study Group).

Lugar

Habitantes de Ommord, Rotterdam, Países Bajos.

Participantes

Un total de 6.989 sujetos mayores de 55 años al inicio del estudio fueron incluidos luego de determinar que no padecían trastornos cognitivos. Se realizó un seguimiento por 8 años (1991-1998), con exámenes para detectar demencia en 1993-1994 y en 1997-1999. Se recolectó información del uso AINES y otros fármacos.

El uso de AINES y otros fármacos fue extraído del registro de las farmacias. Se definieron 4 categorías mutuamente excluyentes en cuanto al uso de AINES: 1) no uso de AINES, 2) corto uso de AINES (1 mes o menos de uso acumulativo), 3) uso intermedio de AINES (más de 1 mes pero < de 24 meses) y 4) uso prolongado (más de 24 meses de uso).

Fuente

\section{Comentario}

Este es un importante estudio que nos ayuda a clarificar conflictos de estudios previos que analizaban los potenciales efectos neuroprotectores de los AINES, tanto para la EA y la DV. La EA es un gigante epidemiológico para todos los que trabajamos con la tercera edad. Afecta a más de 15 millones de personas en todo el mundo, y debido a su escasa letalidad (la sobrevida a la década de realizado el diagnóstico es habitual) la prevalencia oscila desde el $3 \%$ a los 65 años hasta el $47 \%$ a los 85 años de edad. ${ }^{16}$ Clínicamente se expresa por un comienzo insidioso con una posterior y progresiva pérdida de la memoria y de las funciones cognitivas. Anatómicamente se caracteriza por la pérdida de neuronas y de sinapsis en la corteza de hipocampo y luego en otras áreas de la neocorteza cerebral. La destrucción celular ocurre por la acumulación de placas seniles extracelulares (beta amielodes) y ovillos neurofibrilares intraneuronales. Cuando el nivel de pérdida de masa neuronal sobrepasa las capacidades compensatorias de otros circuitos cerebrales, se presentan los síntomas. Para poder entender la plausibilidad biológica de la posible prevención de la aparición de la enfermedad por el uso de AINES, es necesario reseñar brevemente algunos nuevos conocimientos sobre la fisiopatología de la EA. La teoría de la cascada amiloide, avalada parcialmente por evidencia experimental, propone una serie escalonada de eventos en la patogénesis de esta enfermedad que conlleva a la acumulación extracelular del -amiloide. Básicamente la proteína estructural de la membrana plasmática neuronal llamada APP (amyloid precursor protein) empieza a ser degradada por una vía metabólica alternativa produciendo fragmentos de -amiloide, insolubles y neurotóxicos.

En condiciones normales esta vía alternativa degrada una proporción mínima de APP, pero la acción de mutaciones genéticas o factores de riesgo ambientales parecen aumentar la actividad de esta vía catabólica alternativa. La acumulación extracelular de beta-amiloide tendría efecto tóxico neuronal, primero alterando el funcionamiento y finalmente produciendo la muerte celular. Esta neurotoxicidad parece estar mediada por mecanismos inflamatorios y de estrés oxidativo. Este meticuloso estudio ( $p$. ej. el uso de AINEs fue documentado por medio de los registros de farmacia) nos provee evidencia de que existe una asociación inversa

\section{Resultados Principales}

Durante un seguimiento de 6.8 años, desarrollaron demencia 394 sujetos de los cuales 293 reunían criterios de EA, 56 de DV y 45 otros tipos de demencia.

El riesgo no mostró variaciones con respecto a la edad, y el uso de AINES no se asoció con la reducción de aparición de DV (Tabla 1). Tabla 1

\begin{tabular}{|c|c|c|c|c|}
\hline Exposición a AINES & $\begin{array}{c}\text { Sujetos con } \\
\text { demencia } \\
\mathrm{N}=(394)\end{array}$ & $\begin{array}{c}\text { Cohorte entera } \\
\mathrm{N}=(6989)\end{array}$ & $\begin{array}{c}\text { RR }(95 \% \text { IC) } \\
\text { Enf. de ALZHEIMER }\end{array}$ & $\begin{array}{c}\text { RR }(95 \% \text { IC) } \\
\text { Dementia Vascular }\end{array}$ \\
\hline \multicolumn{5}{|l|}{ AINES } \\
\hline No exposición & 210 & 2553 & 1.00 & $1.25(0.63-2.53)$ \\
\hline$<1 \mathrm{mes}$ & 88 & 2001 & $0.95(0.70-1.29)$ & $1.36(0.70-2.64)$ \\
\hline$>1$ a 23 meses & 93 & 2202 & $0.83(0.62-1.11)$ & $0.01^{*}$ \\
\hline$>24$ meses & 1 & 233 & $0.20(0.005-0.83)$ & $0.99(0.13-7.58)$ \\
\hline \multicolumn{5}{|l|}{ Salicilatos orales } \\
\hline No exposición & 252 & 4675 & 1.00 & 1.00 \\
\hline$<1$ mes & 7 & 285 & $0.76(0.31-1.84)$ & - \\
\hline$>1$ a 23 meses & 93 & 1017 & $1.30(0.97-1.74)$ & $2.99(1.57-5.71)$ \\
\hline$>24$ meses & 42 & 1012 & $0.76(0.49-1.19)$ & $4.88(2.38-10.0)$ \\
\hline
\end{tabular}

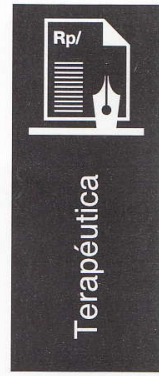

entre el uso crónico de AINEs y la aparición de EA. Además posee como fuerza metodológica, teniendo siempre como limitante el hecho de ser un estudio observacional*, el haber seguido a 7000 pacientes por un período de casi ocho años. Los resultados muestran una relación lineal con respecto al tiempo de duración del uso de AINEs y prevención de aparición de EA, llegando a un riesgo relativo (RR) de 0.20 para aquellos que usaron AINEs por lo menos por dos años (ver tabla 1).

Existen estudios previos que son controversiales con respecto a la prevención de la aparición y el uso de AINEs ${ }^{2,3,4}$. No obstante como expresa el comentario editorial realizado por Breitner y Zandi en la misma publicación solo el estudio de Baltimore mostró resultados similares. Aparentemente son los únicos estudios que evalúan y registran el uso de AINES al menos dos años antes del desarrollo de síntomas. Denotando de esta manera la existencia de un periodo crítico en que durante el cual la exposición a AINEs podría proteger de la aparición de EA. Como dato interesante es de aclarar que los fármacos involucrados han sido diclofenac en un $43 \%$, ibuprofeno en un $21 \%$ y naproxeno en un $17 \%$ llevándose estas tres drogas el $81 \%$ de los fármacos utilizados. Esto podría tener el confundidor* del uso no registrado de aspirina, ya que es de venta libre. No obstante la exposición debería ser la misma para toda la población por el tipo de análisis realizado (análisis multivariable*, ajustando* por comorbilidad y todos los fármacos). Otro dato interesante es que encontraron que estos efectos ocurren tanto en bajas como en altas dosis. un dato no menos interesante es el no hallazgo de asociación con demencia vascular (aunque esta es más infrecuente y por ende de más difícil estudio).

Conclusiones del comentador: Para indicar un tratamiento masivo en población sana es necesario contar con evidencia de estudios controlados aleatorizados. El presente es un interesante estudio observacional ${ }^{\star}$ que plantea la asociación entre el uso de AINEs y la reducción del riesgo de Enfermedad de Alzheimer. Un trabajo que tal vez podrá responder esta pregunta esta siendo realizado por el instituto nacional del Envejecimiento en EE.UU. (www.2stopAD.org).

Por otro lado, el dato negativo de que el uso de AINES no previno la aparición de DV no es menos relevante.

\footnotetext{
Dr. Manuel Montero-Odasso [ Servicio de Clínica Médica y Sección Geriatría. Hospital Italiano de Buenos Aires ]

*Ver glosario

Referencias

1. Mayeux, R and Sano, M. Treatment of Alzheimer's Disease. N EngJ Med 1999; 34: 1670-1679

2. McGeer PL, Schulzer M, McGeer EG. Arthritis and anti-inflammatory agents as possible protective factors for Alzheimer's disease: a review of 17 epidemiologic studies. Neurology 1996; 47: 425-432.

3. Beard CM, Waring SC, O'Brien PC, Kurland LT, Kokmen E. Nonsteroidal anti-inflammatory drug use and Alzheimer's disease: a case-control study in Rochester, Minnesota, 1980 through 1984. Mayo Clin Proc 1998;73: 951-955.

4. Stewart WF, Kawas C, Corrada M, Metter EJ. Risk of Alzheimer's disease and duration of NSAID use. Neurology 1997; 48: 626-632.

5. Aisen PS, Davis KL, Berg JD, et al. A randomized controlled trial of prednisone in Alzheimer's disease. Neurology 2000; 54: 588-593.

6. Herbert LE, Scherr PA, Beckett LA, et al. Age -specific incidence ogf Alzheimer's disease in a comunity popuklation. JAMA; 1995; 273: 1354-1359.
} 